American Journal of Applied Sciences 5 (3): 270-275, 2008

ISSN 1546-9239

(C) 2008 Science Publications

\title{
An Intelligent Routing Algorithm
}

\author{
Amer Nizar Abu Ali \\ Philadelphia University, P.O.Box.1, Amman-19392, JORDAN
}

\begin{abstract}
In this paper we propose a new method of routing in network. The method is based on vague set theory. Our routing method is both static and dynamic in nature and very frequent exchange of routing information is avoided in order to make the network faster. The approach method reduces to a method of fuzzy routing as a special case. The fuzzy routing so developed is different from the other fuzzy routing algorithms.
\end{abstract}

Keywords: Fuzzy Set, Vague Set, Generated Vector (GV), Vague Vector (VV), Most Expected Object (MEO), Most Expected Vague Vector

\section{INTRODUCTION}

One of the key design objectives of B-ISDN is that the provision of a wide range of services to a broadband variety of users utilizing a limited set of connection types and multi-purpose user-network interfaces. Broadband ISDN (B-ISDN) was envisioned as a provider of higher bit rates to the user than $\mathrm{N}$ ISDN. The two prominent enabling technologies for the deployment of B-ISDN are fiber optics and ATM network architecture. ATM refers to switching and multiplexing techniques. As ATM has to support wide range services whose requirements vary over a wide range, the transport of cells must be at high speed. This calls for minimizing the processing time at the intermediate devices like router and the efficient methods for traffic management. Routing is an important functional aspect of networks to transport packets in general (or cells in ATM networks) from source to destination. A router sets up optimized paths among the different nodes in the network. An optimized path is that one which gives low mean packet delay and high network throughput. Many routing algorithms exist in the literature. All these can be broadly classified into static and dynamic algorithms. Dynamic routing algorithms make decision regarding the optimized paths independently of other routers based on the information exchanged among the adjacent routers. This exchange of routing information is carried out periodically increasing the traffic on the network. Most of our traditional tools for formal modeling, reasoning and computing are crisp and precise. But real life data are not always crisp, and all descriptions can not be always expressed or measured precisely. To deal with such type of real life problems, [19] proposed a new mathematical model known as Fuzzy Set Theory. The genuine necessity of such a new mathematical model stem from the fuzziness of natural phenomenon. Fuzzy sets have been applied in wide variety of fields like Computer Science, Medical Science, Management Science, Social Science, Engineering etc. to list a few only. Let $\mathrm{U}$ be a universe of discourse. A fuzzy set A is a class of objects of $U$ along with a membership function $\mu \mathrm{A}$. The grade of membership of $u(u € U)$ in the universe $U$ is 1 , but the grade of membership of $u$ in a fuzzy subset $A$ (of $U$ ) is a real number in $[0,1]$ denoted by $\mu \mathrm{A}(\mathrm{u})$, which signifies that $\mathrm{u}$ is a member of the fuzzy set A up to certain extent, the degree of membership could be zero or more and at most full (i.e. 1). The greater $\mu \mathrm{A}(\mathrm{u})$, the greater is the truth of the statement that 'the element $\mathrm{u}$ belongs to the set A'. Different authors ${ }^{[4],[10],[14]}$ from time to time have made a number of generalizations of Zadeh's fuzzy set theory. Of these, the notion of Vague Set (VS) theory recently reported in IEEE ${ }^{[10]}$ is of interest to us. In most cases of judgments or estimation-procedures, evaluation is done by human beings (or by an intelligent agent) where there certainly is a limitation of knowledge or intellectual functionaries. Naturally, every decisionmaker hesitates more or less, on every evaluation activity. To judge whether a patient has cancer or not, a doctor (the decision-maker) will hesitate because of the fact that a fraction of evaluation he thinks in favour of truthness, another fraction in favour of falseness and rest part remains undecided to him. This is the breaking philosophy in the notion of vague set theory recently reported in IEEE ${ }^{[10]}$

\section{EXISTING ROUTING ALGORITHMS}

Routing is an important issue to communicate among the users on different networks. In today's Internet world, information, split into small blocks called packets or cells, is moved across some kind of 
networks and terminates at destination point. In this process, a data packet passes through a route or path identified by routers. The selection of optimized path between sender and receiver is a major design area of network layer of ISO's OSI reference model ${ }^{[1],[8]}$. Many algorithms for routing are available in the literature which falls in one of two major groups: nonadaptive and adaptive. Non-adaptive (also called static) algorithms do not base their routing decisions on measurements or estimates of the current traffic and topology. Adaptive (also called dynamic) algorithms, in contrast, change their routing decisions to reflect changes in the topology and traffic. These adaptive algorithms decide the routing path based on the information they get from other routers. The various adaptive algorithms, available in the literature, differ in the way they get information (locally, from adjacent routers or from all routers), when they change the routes (e.g. every t $\mathrm{msec}$, when the load changes, or when the topology changes), and what parameter is used for optimization (e.g. distance, number of hops, or estimated transit time, reliability, bandwidth, load etc). These adaptive routing algorithms are much applicable in the present scenario of changing networks both in size and service requirements. Dynamic routing algorithms exchange routing information periodically among the adjacent routers. This is called periodic updates. This period typically ranges from few tens of milliseconds to 1 or 2 minutes. If the updates are too frequent, congestion may occur. On the other hand, if updates are too infrequent, routing may not be efficient. Hence these dynamic algorithms add extra traffic due to the exchange of routing information among the routers to the network. Traffic due to ever increasing demand of new services is also growing. This is making the traffic (due to routing and user information) management issue a complex one and hence it is becoming a major field of research in present days networks like ATM. Distance Vector Routing algorithm, originally proposed by ${ }^{[2],[9]}$ is considered as conventional dynamic routing algorithm as it is being employed widely in today's networks like Routing Information Protocol (RIP) for IP, Cisco's Internet Gateway Routing Protocol (IGRP), AppleTalk's Routing Table Maintenance Protocol (RTMP), etc. In Distance Vector Routing, each router maintains a routing table containing one entry for each destination in the network. This entry tells the preferred outgoing line to use for that destination. The router knows the "distance" (number of hops, queue length or delay) to each of its neighbors. For example, consider that delay is used as a metric and assume that the router knows the delay to each of its neighbors. Once every $t$ msec, each router sends to each neighbor a list of its estimated delays to each destination. It also receives a similar list from each neighbors. Based on this information, a router can find out which estimate seems the best and updates its routing table. This routing table will be used by the router to route the packets for next $\mathrm{T}$ msec $\quad(\mathrm{T}$ $>$ t), after which routing information will be exchanged again and this procedure is repeated. Thus for every $t \mathrm{msec}$, routing information will be exchanged among the adjacent routers which leads to increased traffic on the network. But the advantage of the algorithm is that it updates routing information dynamically for every fixed time interval. We propose a new method, using modern and appropriate mathematical tools of recent births, to reduce the traffic due to exchange of routing information in Distance Vector Routing algorithm retaining its existing advantages.

\section{VAGUE SETS}

In this section, we present some basic preliminaries on the theory of vague sets (VS) recently reported ${ }^{[10]}$ in IEEE. Let $\mathrm{U}=\{\mathrm{u} 1, \mathrm{u} 2 \ldots \ldots \ldots$, un $\}$ be the universe of discourse. The membership function for fuzzy sets can take any value from the closed interval $[0,1]$. Fuzzy set $\mathrm{A}$ is defined as the set of ordered pairs $\mathrm{A}=\{(\mathrm{u}, \mu \mathrm{A}(\mathrm{u})): \mathrm{u} € \mathrm{U}\}$, where $\mu \mathrm{A}(\mathrm{u})$ is the grade of membership of element $u$ in set $A$. The greater $\mu \mathrm{A}(\mathrm{u})$, the greater is the truth of the statement that 'the element $\mathrm{u}$ belongs to the set $\mathrm{A}^{\prime} .{ }^{[10]}$ pointed out that this single value combines the 'evidence for $u$ ' and the 'evidence against $u$ '. It does not indicate the 'evidence for $u$ ' and the 'evidence against $u$ ', and it does not also indicate how much there is of each. Consequently, there is a genuine necessity of a different kind of fuzzy sets which could be treated as a generalization of ${ }^{[4],[13],[20]}$.

Definition Vague Set: A vague set (or in short VS) A in the universe of discourse $U$ is characterized by two membership functions given by :

(i) a truth membership function $\mathrm{tA}: \mathrm{U} \rightarrow[0,1]$ and

(ii) a false membership function $\mathrm{fA}: \mathrm{U} \rightarrow[0,1]$

where $t A(u)$ is a lower bound of the grade of membership of $u$ derived from the 'evidence for $u$ ', and $\mathrm{fA}(\mathrm{u})$ is a lower bound on the negation of $\mathrm{u}$ derived from the 'evidence against $u$ ', and $\mathrm{tA}(\mathrm{u})+\mathrm{fA}(\mathrm{u}) 1$-،. Thus the grade of membership of $u$ in the vague set $A$ is bounded by a subinterval $[\mathrm{tA}(\mathrm{u}), 1-\mathrm{fA}(\mathrm{u})]$ of $[0,1]$. 
This indicates that if the actual grade of membership is $\mu(\mathrm{u})$, then $\mathrm{tA}(\mathrm{u})-6 \mu(\mathrm{u})-61-\mathrm{fA}(\mathrm{u})$. The vague set $A$ is written as $A=\{<u,[t A(u), f A(u)]>: u$ $€ U\}$, where the interval $[\mathrm{tA}(\mathrm{u}), 1-\mathrm{fA}(\mathrm{u})]$ is called the vague value of $\mathrm{u}$ in $\mathrm{A}$ and is denoted by $\mathrm{VA}(\mathrm{u})$. Clearly, an amount of $\pi(\mathrm{x})=1-\mathrm{tA}(\mathrm{x})-\mathrm{fA}(\mathrm{x})$ is still undecided whether and how much of it will cater to truthness as well as falseness. For example, consider an universe $\mathrm{U}=\{\mathrm{DOG}, \mathrm{CAT}, \mathrm{RAT}\}$. Vague set A of $\mathrm{U}$ could be $\mathrm{A}=\{<\mathrm{DOG},[.7, .2]\rangle,<\mathrm{CAT},[.3, .5] .,<\mathrm{RAT},[.4, .6]\rangle\}$. It is worth to mention here that interval-valued fuzzy sets (i-v fuzzy sets) ${ }^{[21]}$ are not vague sets. In i-v fuzzy sets, an interval valued membership value is assigned to each element of the universe considering the 'evidence for u' only, without considering 'evidence against u'. In vague sets both are independently proposed by the decision maker. This makes a major difference in the judgment about the grade of membership.

To explain the definition of a vague set, let us Consider a universe $X=\{x 1, x 2, x 3, x 4\}$. A vague set $A$ of this universe $X$ contains all the elements of $X$, for each element there is a true-membership value in the interval $[0,1]$, and also a false-membership value in the interval $[0,1]$. Thus a vague set in $\mathrm{X}$ will be like below:-

$A=\{(x 1,(t(x 1), f(x 1))),(x 2,(t(x 2), f(x 2))),(x 3$, $(\mathrm{t}(\mathrm{x} 3), \mathrm{f}(\mathrm{x} 3))), \quad(\mathrm{x} 4,(\mathrm{t}(\mathrm{x} 4), \mathrm{f}(\mathrm{x} 4)))\}$.

It means that : $\mathrm{x} 1$ belongs to the vague set $\mathrm{A}$ with truemembership value $t(x 1)$, and false-membership value $\mathrm{f}(\mathrm{x} 1)$, and $\mathrm{x} 2$ belongs to the vague set A with truemembership value $t(x 2)$, and false-membership value $\mathrm{f}(\mathrm{x} 2)$, and $\mathrm{x} 3$ belongs to the vague set A with truemembership value $t(x 3)$, and false-membership value $\mathrm{f}(\mathrm{x} 3)$, and $\mathrm{x} 4$ belongs to the vague set $\mathrm{A}$ with truemembership value $t(x 4)$, and false-membership value $\mathrm{f}(\mathrm{x} 4)$. One universe $\mathrm{X}$ can have infinite number of such vague sets $A, B, C$,

\section{AN INTELLIGENT ROUTING: PROBLEM STATEMENT}

In this paper we propose a new method of routing called by 'vague routing' considering the existing Distance Vector Routing with vague/fuzzy tools. Consider the traffic situation on the roads of a city. During a time interval of $\mathrm{T}$ hours (say 30 minutes), the mean traffic on a road could be assumed to be almost constant (or almost static). During the next 30 minutes, again it is almost constant with another mean value of traffic. So there could be a significant change in these two data of "almost equal". There is no method to know the exact traffic of near future. But the mean-value (of near future) of traffic plays a great role in routing in every network. Thus there is a genuine field of applying fuzzy theory or similar modern tools which can well deal with imprecise data or information. This is the philosophy we will use in our work to find a new method of routing. The vagueness involved in the term almost constant mean of traffic is to be dealt with properly dealt in our research work so that an appropriate application will be successful to propose a new method of routing. We attempt to make our method both dynamic as well as static in nature expecting an increase in the speed of routing as compared to conventional adaptive routers, as we will avoid the very frequent exchange of routing information, without disturbing the routing. This will reduce the computation complexity too at each router of the network.

\section{SOME NEW TERMINOLOGIES}

We first of all develop some theories which will be required to explain our method. We define the new terminologies Generated Vector (GV), Vague Vector (VV), Most Expected Object (MEO), Most Expected Vague Vector (MEVV).

Generated Vector (GV): Consider $\mathrm{k}$ number of $\mathrm{n}$ dimensional vectors $V 1, V 2, \ldots \ldots, V \mathrm{k}$ where

$$
V_{i}=\left[\begin{array}{l}
v_{i 1} \\
v_{i 2} \\
\cdot \\
\cdot \\
v_{i m}
\end{array}\right], i=1,2,3, \ldots, k .
$$

For each $\mathrm{j}(\mathrm{j}=1,2, \ldots, \mathrm{n})$, we will do here $\mathrm{n}$ number of extrapolations by using

Newton's Backward Interpolation formula, for the $\mathrm{n}$ tables given by

\begin{tabular}{cccccc}
\hline 1 & 2 & 3 & 4 & $\ldots \ldots \ldots \ldots \ldots$ & $\mathrm{K}$ \\
$V_{l j}$ & $V_{2 j}$ & $V_{3 j}$ & $V_{4 j}$ & $\ldots \ldots \ldots \ldots \ldots$ & $V_{k j}$ \\
\hline
\end{tabular}

to calculate $v \mathrm{k}+1, \mathrm{j}, \quad \mathrm{j}=1,2,3, \ldots \ldots, \mathrm{n}$. Thus a new vector is generated, which is 


$$
\overline{V_{k+1}}=\left[\begin{array}{c}
\overline{v k+1,1} \\
\hline v_{k+1,2} \\
-\quad-\quad- \\
-\quad-\quad- \\
-\bar{v}-1, n
\end{array}\right]
$$

This vector we may call as a Generated Vector (GV) generated by $\mathrm{V} 1, \mathrm{~V} 2, \ldots, \mathrm{Vk}$.

Vague Vector (VV): By a vague vector (VV)

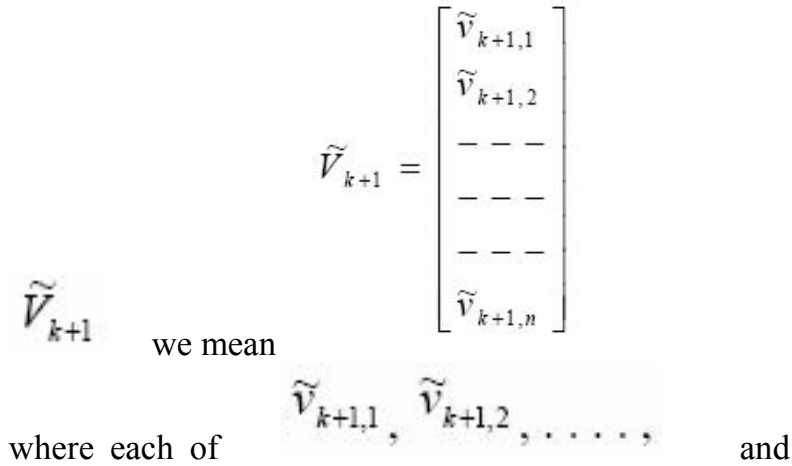

$$
\begin{aligned}
& \widetilde{v}_{k+1, n}
\end{aligned}
$$

is a vague number (A vague number is a vague subset in the. universe of discourse $U$ that is both convex and normal)corresponding to the precise numbers



respectively. We call it a VV generated by the vectors $\mathrm{V} 1, \mathrm{~V} 2, \ldots . ., \mathrm{Vk}$ and denote it by the notation $\mathrm{VV}(\mathrm{V} 1, \mathrm{~V} 2, \ldots, \mathrm{Vk})=V_{1+k}$

Most Expected Object (MEO): Suppose that A is a vague set of a set $X$ with the truth-membership function $t_{A}$ and the false-membership-membership function fA. By the term 'Most Expected Object' (MEO), we mean that element of $\mathrm{X}$ for which the è-value is maximum where the function è is given by

$$
\left\{\begin{aligned}
\theta\left(x_{i}\right) & =\frac{t_{A}\left(x_{i}\right)}{f_{A}\left(x_{i}\right)}, & & \text { if } f_{A}\left(x_{i}\right) \geq \pi_{A}\left(x_{i}\right) \\
& =\frac{t_{A}\left(x_{i}\right)}{\pi_{A}\left(x_{i}\right)}, & & \text { otherwise }
\end{aligned}\right.
$$

Where $\pi(\mathrm{x})=1-\mathrm{tA}(\mathrm{x})-\mathrm{fA}(\mathrm{x})$.

Thus, $\operatorname{MEO}(\mathrm{A})=\mathrm{x}_{\mathrm{q}}$, where $\mathrm{x}_{\mathrm{q}} € \mathrm{X}$ and è $\left(\mathrm{x}_{\mathrm{q}}\right)=\max \{$ è(xi) : xi $\varepsilon \mathrm{X}\}$,

Most Expected Vague Vector (MEVV): Suppose that the $\mathrm{VV}(\mathrm{V} 1, \mathrm{~V} 2, \ldots, \mathrm{Vk})=\widetilde{V}_{k+1}$ where

$$
V_{k+1}=\left[\begin{array}{c}
\tilde{v}_{k+1,1} \\
\tilde{v}_{k+1,2} \\
--- \\
--- \\
--- \\
\widetilde{v}_{k+1, n}
\end{array}\right]
$$

Most Expected Vague Vector (MEVV) of $\widetilde{V}_{k+1}$ is the vector

$$
V_{k+1}=\left[\begin{array}{cc}
\operatorname{MEO} & \left(\tilde{v}_{k+1,1}\right) \\
\operatorname{MEO} & \left(\tilde{v}_{k+1,2}\right) \\
------ \\
----- \\
----- \\
\operatorname{MEO}
\end{array}\right]=\left[\begin{array}{c}
v_{k+1,1} \\
v_{k+1,1} \\
--- \\
--- \\
--- \\
v_{k+1, n}
\end{array}\right],
$$

which we denote by MEVV (V1, V2, ..., Vk).

Vague Routing: Our method is an application of vague theory of Gau and Buehrer (1993) in routing. Suppose that the size of the network is $r$ (the number of routers or nodes). We assume that all the routers are active and the metric is measured by delay.

Consider two choice parameters $\mathrm{n}$ and $\mathrm{T}$ ( to be precisely understood later on).

Method: Once every $\mathrm{t} \mathrm{msec}$, each router sends to each of its neighbor a "list of estimated delay" (LED) to reach the every router of the network. On sending $n$ number of such lists, it halts for T hours. After T hours, the router again sends a fresh set of LED and so on. Clearly each LED is a r-dimensional vector. Our work in this paper presents a method for generating a new type of LED which we call here vague-LED (VLED). For the sake of presentation of our method, let us consider the subnet as shown in Fig.1. 




Fig. 1 A subnet

With no loss of generality consider any router, say F. It has three neighbors A, E and G. Therefore it can receive LED from these three neighbors only. Suppose that at some instant $\hat{o}$, and then at the regular instants $(\hat{o}+t),(\hat{o}+2 t), \ldots \ldots,(\hat{o}+(n-1) t)$, the router $F$ receives the following sets of three LEDs from A, E, and G respectively. LED information received by the router $\mathrm{F}$

\begin{tabular}{|l|l|l|l|}
\hline $\begin{array}{l}\text { At the } \\
\text { instant }\end{array}$ & $\begin{array}{l}\text { LED received } \\
\text { from A }\end{array}$ & $\begin{array}{l}\text { LED received } \\
\text { from E }\end{array}$ & $\begin{array}{l}\text { LED received } \\
\text { from G }\end{array}$ \\
\hline$\tau$ & $V_{0}^{A}$ & $V_{0}^{E}$ & $V_{0}^{G}$ \\
\hline$\tau+\mathrm{t}$ & $V_{1}^{A}$ & $V_{1}^{E}$ & $V_{1}^{G}$ \\
\hline$\tau+2 \mathrm{t}$ & $V_{2}^{A}$ & $V_{2}^{E}$ & $V_{2}^{G}$ \\
\hline$\ldots$ & $\ldots$ & $\ldots$ & $\cdots$ \\
$\ldots$ & $\ldots$ & $\cdots$ & $\ldots$ \\
$\ldots$ & $\ldots$ & $\ldots$ & $\ldots$ \\
$\ldots$ & $\ldots$ & $\cdots$ & $\cdots$ \\
$\ldots$ & $\ldots$ & $\ldots$ & $\ldots$ \\
\hline$\tau+(\mathrm{n}-1) \mathrm{t}$ & $V_{n-1}^{A}$ & $V_{n-1}^{E}$ & $V_{n-1}^{G}$ \\
\hline
\end{tabular}

Each entry vector in the above table is r-dimensional (in this example $r=12$ ).For example (hypothetical), if

$$
V_{3}^{C}=\left[\begin{array}{l}
x_{1} \\
x_{2} \\
\cdot \\
\cdot \\
\cdot \\
x_{r}
\end{array}\right],
$$

then it means that the optimal delay from $\mathrm{C}$ to the router $\mathrm{R} 1$ is $\mathrm{x} 1$, the optimal delay from $\mathrm{C}$ to the router $\mathrm{R} 2$ is $\mathrm{x} 2$, and so on.

Now compute the following three vectors:

$$
\begin{aligned}
& \text { (1) } \operatorname{MEVV}\left(V_{0}^{A}, V_{1}^{A}, \ldots \ldots, V_{n-1}^{A}\right)=V_{n}^{A}, \\
& \text { (2) } \operatorname{MEVV} \quad\left(V_{0}^{E}, V_{1}^{E}, \ldots \ldots, V_{n-1}^{E}\right)=V_{n}^{E}, \\
& \text { (3) } \operatorname{MEVV} \quad\left(V_{0}^{G}, V_{1}^{G}, \ldots \ldots, V_{n-1}^{G}\right)=V_{n}^{G},
\end{aligned}
$$

The collection of these three vectors is called vagueLED ( VLED ). During the computation of these three vectors, the router $\mathrm{F}$ will be functioning according to the previous VLED. The new VLED now will remain valid to the router $\mathrm{F}$ for $\mathrm{T}$ hours next, until the next VLED is computed. With the help of this VLED, routing will be done by F. Once an VLED is computed, the previous VLED gets replaced. In this way the routing will be continued at every router of the net.

Benefits of using vague theory: If we use the vague theory, then the new theories/results will be obtained in vague notions. All these results can be reduced to fuzzy also as a special case. If we use fuzzy theory instead of crisp mathematics, we will get better results in case of the problem under studied. Similarly, if we use vague theory instead of fuzzy theory, we will get further better results. The only demerit is that theoretical complexity of soft computation with vague theory will be more, and also more will be the execution time accordingly. But in many cases we require more and more accurate results at the cost of time and any Complex mathematics.

\section{CONCLUSION}

The work done in this paper is an application of vague theory of Gau and Buehrer (1993) in network. We have proposed a new method of routing called 'vague routing'. The method could be called an intelligent method because of its capability to handle uncertainty in traffic management by applying vague theory. Actually, we have considered Distance Vector Routing with vague tools. Consider the traffic situation on the roads of a city. During a time interval of $\mathrm{T}$ hours (say 30 minutes), the mean traffic on a road could be assumed to be almost equal. During the next 30 minutes, again it is almost equal with another mean 
value of traffic. This is the philosophy we have used in this work. The vagueness in the value of almost equal mean of traffic is to be dealt with proper truthmembership function and false-membership function. The method proposed is both dynamic as well as static. The method does not need continuous resource management traffic, but it works on updated information (updated every $\mathrm{T}$ hours). Thus a gain in the reduction of resource management traffic has been made possible. It is claimed that the method will improve the overall performance of the network. The disadvantage of the method is that in case a catastrophic traffic of packets arises in any path, the method may not yield better performance.

The work for the construction of truthmembership and false-membership functions for generating VLED in vague routing needs a rigorous study and survey, which is under our next research plan. If fortunately, all the indeterministic functions are null functions (i.e. if there is no hesitation), vagueness reduces to fuzziness and then the vague routing will be reduced to 'fuzzy routing' as a special case. The fuzzy routing reported by the authors ${ }^{[15],[17]}$ in queuing systems and computer network routing are different from the fuzzy routing proposed by us in this work.

\section{REFERENCES}

1. S. Tanenbaum., 1988.Computer Networks, Prentice-Hall, Englewood Cliffs, NJ

2. Bellman, R.E., Dynamic, 1957. Programming, Princeton,NJ; Princeton University Press, 1957

3. Chen, Shyi-Ming, 2003. Analyzing fuzzy system reliability using vague set theory, Int. Jou. of Applied Sc. \& Engg. Vol.1,pp:82-88

4. Dubois and Prade, 1990.Fuzzy Sets \& Systems: Theory and Applications, Academic Press, New York

5. Dubois, D. and Prade, H., Toll sets and toll logic. In Fuzzy Logic : State of the art, R. Lowen and M. Roubens, eds., Dordrecht : Kluwer Aca. Publisher

6. Dubois, D. and Prade, H.,1987. Twofold fuzzy sets and rough sets : some issues in knowledge representation. Fuzzy sets and Systems. Pp: 3-18

7. Dubois, D. and Prade, H.,1989. Rough fuzzy sets and fuzzy rough sets, Int. Jou.Gen Sys. Pp:191-209
8. D. Driankov, H. Hellendoorn and M. Reinfrank, An Introduction to Fuzzy Control. 1993. SpringerVerlag, Berlin, New York

9. Ford,L.R. and Fulkerson,D.R., 1962. Flows in Networks, Princeton,NJ; Princeton University Press

10. Gau, W. L. and Buehrer, D, J.,1993. Vague sets, IEEE Transactions on Systems, Man and Cybernetics, Vol.23, pp: 610-614

11. Goguen, J. A., L- fuzzy sets, Jou. Maths. Anal. Appl. 18 (1967) 145-174

12. Hirota, K., 1981. Concepts of probabilistic sets, Fuzzy Sets and Systems. 5(1) 31-46

13. Kaufmann, A, Introduction to the Theory of Fuzzy Subsets, 1975. Academic Press,New York,

14. Mizumoto, M. and Tanaka, K.,1976. Some properties of fuzzy sets of type 2. Info.and Control. Vol(31).pp:312- 340

15. Runtong Zhang and Yannis A. Phillis, 1997. "Fuzzy Routing of Queueing Systemswith Heterogeneous Servers", Proc. of IEEE Intl. Conference on Robotics and Automation Albuquerque, New Mexico, pp. 2340-2345

16. Runtong Zhang and Yannis A. Phillis,1997. "Fuzzy Service Rate Control of Queueing Systems", Man and Cybernetics on Information, Intelligence and Systems (IEEE/SMC'96), October 14-17, 1996, Beijing, China

17. Sridhar Pithani and Adarshpal S. Sethi, 1993. "A fuzzy Set Delay Representation for Computer Network Routing Algorithms" Proc. Second Intl. Symposium on Uncertainty Modeling and Analysis, College Park, MD), pp.286-293

18. Wang, Jue., Liu, San-Yang., and Zhang, Jie.,2005, Roughness of a vague set, Int.Jou. of Comp. Cognition. Vol.3(3)pp:82-86

19. Zadeh, L.A,2005. Fuzzy sets, Info. and Control. 8, pp: 338-353

20. Zadeh, L.A.,1975. The concept of a linguistic variable and its application to approximate reasoning-I, Info. And Control.8,pp:199-249

21. Zimmermann, H. J., 1991. Fuzzy Set Theory and Its Applications, Kluwer cademic Publishers, Boston, USA, 